\title{
Niveles de actividad física en alumnado de Educación Primaria de la provincia de Granada Physical activity levels of Primary Education students in Granada \\ Félix Zurita-Ortega, JoséL Luis Ubago-Jiménez, Pilar Puertas-Molero, Gabriel González-Valero, Manuel Castro-Sánchez, Ramón Chacón-Cuberos \\ Universidad de Granada (España)
}

Resumen. La actividad física se ha convertido en uno de los pilares básicos de nuestra vida, mejorando nuestra salud física, mental y social. Por esta razón, es necesario que desde la Educación Primaria se creen hábitos hacia la práctica deportiva. El presente estudio tiene como objetivos: a) describir los niveles de actividad física en el alumnado de $5^{\circ}$ y $6^{\circ}$ curso por género y, b) relacionar las variables psicosociales con la práctica de actividad física, género y académicas. Para su realización hemos utilizado una muestra de 160 alumnos de entre 10 y 12 años (M=11,72) del área metropolitana de Granada. Para la recogida de datos nos hemos servido del cuestionario de actividad física (PAQ-A), además de una hoja de registro para determinar el género, si son repetidores, si están federados y, si los padres practican actividad física. Utilizando, para el análisis de datos, el programa estadístico SPSS versión 22.0. A grandes rasgos, los resultados muestran que los niños realizan más actividad física que las niñas, que los jóvenes federados realizan más actividad física que los no federados y que los alumnos con padres que realizan actividad física tienen una mayor predisposición hacia la práctica deportiva.

Palabras clave. Actividad física, PAQ-A, Educación Primaria.

Abstract. Physical activity has become one of the most important pillars of our life, improving our physical, mental and social health. For that reason, it is necessary to create habits of sport practice from primary education. The objectives of the present study are: a) to describe the levels of physical activity in $5^{\text {th }}$ - and $6^{\text {th }}$-year students by gender, and b) to relate psychosocial variables to physical activity, gender, and academic activity. A total of 160 students between 10 and 12 years from Primary Education centres in Granada participated in this research. For the data collection, we used the physical activity questionnaire for adults (PAQ-A). In addition we used a registration sheet to determine students' gender, if they are repeaters, if they are federated, and their parents' engagement in physical activity. Data analysis was performed using the statistical program SPSS 22.0. Results show that boys perform more physical activity than girls. Also, children who are federated do more physical activity than those non-federated. Students whose parents practice physical activity have a greater predisposition towards sports practice.

Keywords. Physical Activity, PAQ-A, Primary Education.

\section{Introducción}

La continua práctica de actividad física es un aliciente constante y cotidiano en nuestra vida debido a los múltiples beneficios en la salud física y mental que nos produce(Merino \& González, 2006). De hecho, estudios como el de Oviedo, Sánchez, Castro, Calvo, Sevilla, Iglesias, et al. (2013), han relacionado la falta de actividad física y el sedentarismo con problemas de salud.

Además de los beneficios estéticos, el ejercicio físico reporta una amplia variedad de mejoras sobre la salud. El correcto desarrollo de huesos, músculos y articulaciones, así como la tenencia de un sistema cardiovascular saludable y ayudar a tener un peso óptimo son solo una muestra de ello. Deigual modo, es un claro regulador de la autoconfianza y la interacción social, fortaleciendo el bienestar personal, social y emocional (Reverter-Masià, Plaza-Montero, Jové, \& Hernández-González, 2014; Puertas-Molero, González-Valero \& Sánchez-Zafra, 2017 y Ubago-Jiménez, Viciana-Garófano, Pérez-Cortés, Martínez-Martínez, Padial-Ruz \& Puertas-Molero, 2018). A su vez, autores como GonzálezHernández y Portolés-Ariño, (2016) señalan una relación positiva entre la actividad física y el rendimiento académico, tiendo en cuenta como el repetir curso influye directamente en la adherencia a la práctica de actividad física.

La actividad física para Sánchez Bañuelos (1996) es contemplada como el movimiento corporal de cualquier tipo, producido por la contracción muscular y que conduce a un incremento sustancial del gasto energético del individuo que la realiza. Por otro lado, para la Organización Mundial de la Salud (2010), supone realizar cualquier tipo de movimiento corporal que sea producido por el aparato muscular y, que conlleve un consumo de energía. La actividad física, por tanto, engloba el ejercicio, así como otras actividades que suponen un movimiento corporal.Algunos ejemplos de ello son el juego, el trabajo, las formas de transporte activas (bicicleta, patines, etc.), las tareas domésticas o las actividades de ocio y tiempo libre.

El abandono o ausencia de la práctica de actividad física es un

Fecha recepción: 21-09-17. Fecha de aceptación: 25-01-18 José Luis Ubago-Jiménez jlubago@ugr.es suceso bastante complejo, ya que puede darse el caso de que el niño/a deje de practicar de manera temporal o que cambie de actividad física a practicar, por lo cual no podríamos considerarlo como abandono global. Cabe destacar que los jóvenes tienen muchas opciones a la hora de ocupar su tiempo libre, por lo que a veces no se produce el abandono de la actividad por falta de motivación hacia el deporte, sino que se pierde el interés por otra actividad que la sustituye (Granda, Montilla, Barbero, Mingorance \& Alemany, 2010; Macarro, Romero \& Torres, 2010).

Una de las grandes inquietudes entre los docentes de educación física es el abandono de la práctica deportiva que se produce entre el alumnado al pasar de la niñez a la adolescencia (Serra, Generelo \& Zaragoza, 2010; Dionne \& Parent, 2015; Castro-Sánchez, Zurita-Ortega, Martínez-Martínez, Chacón-Cuberos \& Espejo-Garcés, 2016). La actividad física escolar merece recibir la adecuada importancia ya que se ha convertido en el único emplazamiento en el cual los niños tienen la oportunidad de realizar algún tipo de actividad física o práctica de cualquier deporte (Martínez, Aznar, \& Contreras, 2015). La asignatura de educación física cuenta con una carga lectiva bastante limitada dentro del currículum en educación primaria, por lo cual, será muy complicado crear omanteneruna vida físicamente activapara los alumnos (Rodríguez, García-Cantó, Sánchez-López \& López-Miñarro, 2013; MéndezAlonso, Fernández-Río, Méndez-Giménez \& Prieto-Saborit, 2015).

La sintonía existente entre los conceptos como actividad física y salud hacen que sean términos que caminen paralelamente. Así pues, el abandono, carencia o poca práctica de actividad física en los niños es algo que suele perdurar durante la adolescencia y en la vida adulta (Espejo-Garcés, Lozano-Sánchez \& Fernández-Revelles, 2017) y puede producir enfermedades asociadas al sedentarismo como la obesidad (Zurita-Ortega, Cepero, Ruiz, Linares, Cachón \& Zurita-Molina, 2011; Bartrina, 2013; Espejo-Garcés, Cabrera-Fernández, Castro-Sánchez, López-Fernández, Zurita-Ortega \& Chacón-Cuberos, 2015). Este hecho denota como las generaciones actuales son menos activas que las generaciones de sus progenitores (Leite, Carvalho de Mesquita \& Dias, 2015; Zurita-Ortega, Castro-Sánchez, Álvaro-González, RodríguezFernández \& Pérez-Cortés, 2016), llegando a reducir el consumo energético en, aproximadamente, $600 \mathrm{kcal} /$ día respecto a las anteriores generaciones (Boreham \& Ridoch, 2001; Rodríguez, García-Cantó, SánchezLópez \& López-Miñarro, 2013). 
Estudios como los de Klee, Tavares, Horta, Gelatti y Santana (2004), Marcellini, Perera, Rodhain y Ferez, (2016) y González, Zurita, Puertas, Espejo, Chacón y Castro, (2017) abordan el abandono de la actividad física en la etapa de la educación primaria, afirmando el incremento, en las últimas décadas, del sedentarismo entre la población infantil. Este hecho refleja como el $45 \%$ de los niños no realiza ningún tipo de deporte fuera del horario escolar (Isorna, Rial \& Vaquero, 2014). Igualmente, declaran que durante la etapa escolar se produce un aumento significativo de la actividad física, pero, en el tránsito hacia el instituto, los chicos se decantan por un deporte en concreto, practicándolo con más asiduidad, mientras que las chicas prefieren realizar otro tipo de actividades (Serra Puyal et al, 2010; Dionne \& Parent, 2015).

El aumento del sedentarismo, en los últimos años, a causa de los videojuegos, internet y las horas de televisión (Chacón-Cuberos, Zurita-Ortega, Castro-Sánchez, Espejo-Garcés, Martínez-Martínez \& PérezCortés, 2017), ha acarreado que los individuos dejen progresivamente de realizar actividades físicas, practicar algún deporte o simplemente jugar al aire libre (Arufe-Giráldez, Chacón-Cuberos, Zurita-Ortega, LaraSánchez \& Castro-García, 2017). En este sentido, la concienciación de los jóvenes sobre la relación entre la actividad física y una vida saludable se convierte en uno de los objetivos más acuciantes, ya que, gracias a ella, mantendremos un cuerpo sano, evitando el aumento de los niveles de obesidad y otros trastornos médicos (Zurita-Ortega \& ÁlvaroGonzález, 2014; Ambroa De Frutos, 2016; Álvaro-González, ZuritaOrtega, Viciana-Garófano, Martínez-Martínez, García-Sánchez \& Estévez-Díaz, 2016 y González-Valero, Zurita-Ortega, Puertas-Molero, Chacón-Cuberos, Espejo- Garcés \& Castro-Sánchez, 2017).

Por ello, el principal objetivo del presente estudio es determinar la relación entre las variables psicosociales con la práctica de actividad física, el género y las variables académicas. Extrayéndose de la revisión literaria las relaciones existentes entre las variables de estudio.

\section{Método}

\section{Diseñoy participantes}

Se realizó un estudio de carácter cuantitativo-descriptivo y de corte transversal con una muestra representativa de 160 escolares que cursaban $5^{\circ}$ y $6^{\circ}$ curso de Educación Primaria del área metropolitana de Granada con edades comprendidas entre los 10 y 12 años de edad $(\mathrm{M}=11,72 ; \mathrm{DT}=0,500)$. De la muestra total, 71 eran niños $(44,4 \%)$ y 89 niñas (55,6\%).

Tabla 1.

\begin{tabular}{|c|c|c|c|c|}
\hline & \multicolumn{2}{|c|}{ Frecuencia } & Sí & No \\
\hline Actividad Física & $\begin{array}{l}\text { Baja } \\
\text { Media } \\
\text { Alta }\end{array}$ & $\begin{array}{c}48(30 \%) \\
83(51,9 \%) \\
29(18,1 \%)\end{array}$ & & \\
\hline $\begin{array}{c}\text { Repetidor } \\
\text { Actividad Física padres }\end{array}$ & & & $\begin{array}{c}7(4,4 \%) \\
127(79,4 \%)\end{array}$ & $\begin{array}{l}153(95,6 \%) \\
33(20,6 \%)\end{array}$ \\
\hline Federado & & & $70(43,8 \%)$ & $90(56,3 \%)$ \\
\hline
\end{tabular}

Instrumentos y variables

Las variables utilizadas en este estudio han sido las siguientes:

- Género: diferenciando el masculino y femenino.

- Actividad física: está dividida en nivel bajo de actividad física, nivel medio de actividad física y nivel alto de actividad física.

- $\quad$ Repetidor: señalando los repetidores y no repetidores.

- Actividad Física en los padres: dividiéndose en la práctica o no de los padres de actividad física.

- Federado: observándose si el sujeto se encuentra federado o no.

El análisis de las variables, con la excepción de la actividad física, se ha llevado a cabo mediante una hoja de auto-registro, compuesto de diversas preguntas en las que se recoge la información de las variables mencionadas anteriormente.

El cuestionario empleado para medir la actividad física fue el Physical Activity Questionnaire for Adolescents (PAQ-A) en su versión en castellano. El cuestionario PAQ-A fue diseñado para valorar la actividad física de los adolescentes para su utilización en un estudio de la Universidad de Saskatchewan (Canadá), elaborado por Kowalski, Crocker y
Faulkner (1997). El cuestionario está formado por nueve preguntas valorando distintos aspectos de la actividad física realizada por el sujeto mediante una escala tipo Likert de cinco puntos. Únicamente se utilizan ocho preguntas para el cálculo de la puntuación final. El PAQ-Avalora la actividad física del sujeto durante los últimos siete días en su tiempo libre, durante las clases de educación física, así como en diferentes horarios durante los días de clase (comida, tardes y noches) y durante el fin de semana. Siendo las dos últimas preguntas las que valoran cuál es el nivel de actividad física. Sobre cinco propuestos, los encuestados deben seleccionar cuál es el que mejor describe su grado de actividad física a lo largo de la semana, así como su frecuencia diaria. La puntuación final se obtiene mediante la media aritmética de las puntuaciones obtenidas en estas ocho preguntas. La última pregunta permite conocer si el sujeto estuvo enfermo o existió alguna circunstancia que le impidió realizar actividad física durante esa semana.

\section{Procedimiento}

Se solicitó la colaboración a los centros educativos del área metropolitana de Granada a través de una carta informativa elaborada por el Departamento de Didáctica de la Expresión Musical, Plástica y Corporal de la Facultad de Ciencias de la Educación de la Universidad de Granada. La recogida de datos se realizó en horario lectivo. El proceso transcurrió sin incidencias, contando siempre con la presencia de los investigadores para asegurar una correcta aplicación de los instrumentos descritos, así como para la resolución de posibles dudas. El estudio fue realizado respetando todos los criterios de la Declaración de Helsinki, Finlandia en 1961 (revisada en Hong Kong en 1989 y en Edimburgo, Escocia en 2000), y aprobada por el Comité de Ética del Hospital Puerta de Hierro (Madrid, España) y el Comité de Bioética del Consejo Superior de Investigaciones Científicas (CSIC).

\section{Análisis de datos}

Se han analizado los datos con el paquete de programa estadísticos SPSS ${ }^{\circledR} 22.0$ donde se determinaron los parámetros estadísticos (frecuencias, medias y tablas de contingencia) necesarios para este tipo de estudio. Los estadísticos descriptivos del estudio se muestran como media \pm desviación estándar. Las diferencias entre géneros fueron analizadas mediante análisis de varianza simple (ANOVA). La actividad física, valorada mediante el PAQ-A, fue analizada mediante una correlación no paramétrica de Spearman (rho) para el total de la muestra y diferenciada por género. Para analizar las asociaciones entre la actividad física, estar federado, ser repetidor se utilizó el coeficiente de la correlación de Pearson, estableciendo el nivel de significación en $p<.05$.

\section{Resultados}

La Tabla 2 muestra la relación existente entre el género de los participantes y si están o no federados, revelando diferencias estadísticamente significativas (federados 39 vs 32; no federados 31 vs 58; $p=.011$ ). Estas asociaciones vienen producidas dado que en el género masculino encontramos más número de federados que en el femenino. De 71 niños el 54,9\% (n=39) están federados y el 45,1\% (n=32) no lo están; sin embargo, de 89 niñas sólo un 34,8\% (n=31) de ellas están federadas y el $65,2 \%(n=58)$ no lo están. Del mismo modo, volvemos a encontrar diferencias estadísticamente significativas en relación al género y a la actividad física $(p=.035$ ). Estas diferencias se deben a que los chicos realizan más actividad física que las chicas, puesto que de 71 niños, el 19,7\% (n=14) realizan una actividad física baja; el 57,7\% $(\mathrm{n}=41)$ realiza una actividad física media y el $22,5 \%(\mathrm{n}=16)$ practica una actividad física alta; de 89 niñas, el 38,2\% (n=34) realizan una actividad física baja; el 47,2\% ( $\mathrm{n}=42)$ practica una actividad física media y el $14,6 \%(n=13)$ realiza una actividad física alta.

Tabla 2.

Relación entre género y estar federado

\begin{tabular}{|c|c|c|c|c|c|c|c|}
\hline \multirow{2}{*}{ Género } & \multicolumn{2}{|c|}{ Federado } & \multirow[b]{2}{*}{ Sig. } & \multicolumn{3}{|c|}{ Actividad Física } & \multirow[b]{2}{*}{ Sig. } \\
\hline & Sí & No & & Baja & Media & Alta & \\
\hline Hombre & 39 & 32 & .011 & 14 & 41 & 16 & .035 \\
\hline Mujer & 31 & 58 & .61 & 34 & 42 & 13 & .086 \\
\hline
\end{tabular}


Asimismo, existen diferencias estadísticamente significativas entre estar federado y la práctica de actividad física (federados 39 vs 32; 31vs 58; $p=.000$ ) (Tabla 3), producidas porque de 70 sujetos federados, el $14,3 \%(n=10)$ realiza una actividad física baja; el 58,6\% $(n=41)$ practica una actividad física media y el 27,1\% (n=19) una actividad física alta; de los 90 sujetos no federados, el 42,2\% ( $\mathrm{n}=38$ ) realiza una actividad física baja; el 46,7\% (n=42) una actividad física media y el 11,1\% (n=10) una actividad física alta. Mientras que las diferencias entre estar federado y si los padres practican alguna actividad física ( $p=.001$ ), se observan porque de los 127 participantes que tienen padres que realizan actividad física, el 50,4\% (n=64) están federados y el 49,6\% (n=63) no. Sin embargo, de los 33 sujetos que sus padres no practican actividad física, solo el 18,2\% ( $n=6)$ de ellos están federados y el 81,8\% ( $n=27)$ no lo están.

Tabla 3.

Relación entre estar federado y la práctica de actividad física de sujetos y padres

\begin{tabular}{|c|c|c|c|c|c|c|c|}
\hline \multirow{2}{*}{ Federado } & \multicolumn{2}{|c|}{ Actividad Física padres } & \multirow{2}{*}{ Sig. } & \multicolumn{3}{|c|}{ Actividad Física } & \multirow{2}{*}{ Sig. } \\
\hline & Sí & No & & Baja & Media & Alta & \\
\hline Sí & 39 & 32 & .001 & 10 & 41 & 19 & .000 \\
\hline No & 31 & 58 & .089 & 38 & 42 & 10 & .32 \\
\hline
\end{tabular}

$\frac{\text { No }}{\text { Nota. }}{ }^{* *} p<.01 ; * p<.05$

\section{Discusión}

El presente estudio realizado con estudiantes de quinto y sexto curso de Educación Primaria, tiene como principal objetivo relacionar las variables psicosociales con la práctica de actividad física, el género y las variables académicas. Algunos estudios previos de similares características son los realizados por Blández, Fernández-García y Sierra, (2007), Martínez-Gómez, Martínez-de-Haro, Pozo, Welk, Villagra, Calle, et al. (2009), Granda et al, (2010), Chacón-Cuberos, Arufe, Cachón-Zagalaz, Zagalaz y Castro-García, (2016).

En torno a la variable, de practicar actividad física fuera del centro, un gran número de los encuestados respondieron que sí, coincidiendo con los resultados del estudio de Alonso, Carranza, Rueda y Naranjo, (2014), en el que la actividad física realizada por los sujetos se encuentra por encima de la media establecida por el PAQ-A, (3,23 días en niños y 2,94 para las niñas). También es cierto que la práctica de actividad física disminuye conforme aumenta la edad (Valdés, Godoy, Herrera, Álvarez \& Durán, 2014).

Respecto a la variable estar federado, se ha determinado que los chicos son más propensos a practicar deportes federados respecto a las chicas. Este hallazgo respalda los resultados de otros estudios como los de Molinero, Castro, Ruiz, González, Mora y Márquez, (2010) e Isorna et al, (2014). Al mismo tiempo, también se ha observado una tendencia positiva respecto al número de escolares vinculados a algún club o equipo, debido al auge que están teniendo las empresas que ofrecen estos servicios, en los últimos años.

Focalizando la atención en la variable género, descubrimos que la práctica de actividad física es menos frecuente entre las niñas, produciéndose un prematuro abandono de las adolescentes y mujeres (Douthitt, 1994) de las actividades físico-deportivas. Luego, en cuanto a la variable género, se ha evidenciado como los hombres practican deporte con más asiduidad, que las mujeres. A colación de estos resultados, De Hoyo y Sañudo (2007) revelan en su estudio la incipiente preocupación por los bajos porcentajes de actividad física de las chicas.

Por otro lado, los sujetos que cuentan con un entorno familiar que practica deporte, tienen mayor probabilidad de realizar actividad física, bien sea federada o no (Revuelta \& Esnaola, 2011). Resulta obvio como los padres son un modelo a seguir para sus hijos, estableciendo una relación de causa efecto entre su práctica de actividad física y la de sus hijos (Peiron, \& Ruiz-Juan, 2013; Ruiz-Risueño \& Ruiz-Juan, 2015 y Castro-Sánchez et al, 2016).

En nuestro estudio encontramos con los sujetos que están federados realizan más actividad física que aquellos que no lo están. Demostrándose la relación entre la práctica de actividad física con estar federado (Palou, Ponseti, Gili, Borràs \& Vidal, 2005; Isorna et al, 2014).

Por otro lado, incidiendo en los aspectos que relacionan las activi- dades físicas con el buen rendimiento escolar, la muestra estudiada obtuvo porcentajes elevados entre la práctica de actividades físicas fuera del centro escolar no habiendo repetido curso, revelando, de este modo, los beneficios que aporta la actividad deportiva en aspectos sociales y cognitivos (Bailey, 2006) y una mejora del provecho académico (Pastor, Gil, Tortosa \& Martínez, 2012).

A modo de conclusión, el estudio realizado revela como los chicos tienen mayor tendencia hacia la práctica deportiva tanto si están federados como si no lo están que las chicas. Los sujetos que tienen padres que realizan actividad física tienen mayor probabilidad a realizar actividades físico-deportivas. En línea similar, aquellos sujetos que practican actividad física obtienen mejores resultados académicos.

\section{Referencias}

Alonso, F. J., Carranza, M. D., Rueda, J. D. y Naranjo, J. (2014). Composición corporal en escolares de primaria y su relación con el hábito nutricional y la práctica reglada de actividad deportiva. Revista Andaluza de Medicina del Deporte, 7(4), 137-142. Recuperado de: https://goo.gl/8kSPBE

Álvaro-González, J., Zurita-Ortega, F., Viciana-Garófano, V., MartínezMartínez, A., García-Sánchez, S. y Estévez-Díaz, M. (2016). Actividad física de adolescentes: implicación de sustancias nocivas, modalidad practicada y familia. Psicologia Escolar e Educacional, 20(1), 13-22. Doi: http://dx.doi.org/10.1590/2175-3539/2015/0201908 Recuperado de: https://goo.gl/jRHr3F

Ambroa De Frutos, G. (2016). Impacto del sedentarismo sobre la práctica de actividad física y la salud. Análisis de la situación en España. Revista Española de Educación Física y Deportes, 412, 33-44. Recuperado de: https://goo.gl/c7dhzh

Arufe-Giráldez, V., Chacón-Cuberos, R., Zurita-Ortega, F., Lara-Sánchez, A. y Castro-García, D. (2017). Influencia del tipo de centro en la práctica deportiva y las actividades de tiempo libre de escolares. Revista Electrónica Educare, 21(1), 1-19. Doi: http://dx.doi.org/ 10.15359/ree.21-1.6 Recuperado de: https://goo.gl/yVMZtK

Bailey, R. (2006). Physical Education and Sport in Schools: A Review of Benefits and Outcomes. Journal of School Health, 76, 397-401. Doi: 10.1111/j.1746-1561.2006.00132.x Recuperado de: https:// goo.gl/GtXrwc

Bartrina, J.A. (2013). Public health and the prevention of obesity: Failure or success? Nutrición hospitalaria, 28(5), 128-137. Recuperado de: https://goo.gl/umZmKT

Blández, J., Fernández-García, E. y Sierra, M. A. (2007). Estereotipos de género, actividad física y escuela: la perspectiva del alumnado. Profesorado, 11(2), 1-21. Recuperado de: https://goo.gl/2nmtX4

Boreham, C. y Riddoch. C. (2001). The physical activity, fitness and a health of children. Journal of Sports Sciences, 19, 915-929. Recuperado de: https://goo.gl/remJJy

Castro-Sánchez, M., Zurita-Ortega, F., Martínez-Martínez, A., ChacónCuberos, R. y Espejo-Garcés, T. (2016). Clima motivacional de los adolescentes y su relación con el género, la práctica de actividad física, la modalidad deportiva, la práctica deportiva federada y la actividad física familiar. RICYDE. Revista Internacional de Ciencias del Deporte, 45(12), 262-277. Doi:10.5232/ricyde Recuperado de: https://goo.gl/dPuywh

Chacón-Cuberos, R., Zurita-Ortega, F., Castro-Sánchez, M., Espejo-Garcés, T., Martínez-Martínez, A. y Pérez-Cortés, A. J. (2017). Clima motivacional hacia el deporte y su relación con hábitos de ocio digital sedentario en estudiantes universitarios. Saúde y Sociedade São Paulo, 26(1), 29-39. Doi:10.1590/S0104-12902017166561 Recuperado de: https://goo.gl/QT6ER8

Chacón-Cuberos, R., Arufe, V., Cachón-Zagalaz, J., Zagalaz, M. L. y Castro-García, D. (2016). Estudio relacional de la práctica deportiva en escolares según el género. SPORT TK-Revista EuroAmericana de Ciencias del Deporte, 5(1), 85-92. Recuperado de: https://goo.gl/ $12 \mathrm{FnBs}$

De Hoyo, M. y Sañudo, F.B. (2007). Motivos y hábitos de práctica de actividad física en escolares de 12 a 16 años en una población rural de Sevilla. Revista Internacional de Medicina y Ciencias de la Actividad Física y del Deporte, 26, 2-9. Recuperado de: https://goo.gl/vbX1D3

Dionne, S. y Parent, S. (2015). The factors that influenced the decision to abandon athletics among youth 16 to 19 years: a case study in Quebec. Loisir and societe-society and leisure, 38(2), 285-304. Doi: 10.1080/07053436.2015.1040630 Recuperado de: https:/goo.gl/ 
PLXhk5

Douthitt, V.L. (1994). Psychological determinants of adolescent exercise adherence. Adolescence, 29(115), 711-722. Recuperado de: https:/ goo.gl/KHnQZJ

Espejo-Garcés, T., Cabrera-Fernández, A., Castro-Sánchez, M., LópezFernández, J. F., Zurita-Ortega, F. y Chacón-Cuberos, R. (2015). Modificaciones de la obesidad a través de la implementación de herramientas físico-posturales en escolares. Retos. Nuevas tendencias en Educación Física, Deporte y Recreación, 28, 78-83. Recuperado de: https://goo.gl/1yh4F8

Espejo-Garcés, T., Lozano-Sánchez, A. M. y Fernández-Revelles, A. B. (2017). Revisión sistemática sobre la resiliencia como factor influyente en el transcurso de la etapa adolescente. ESHPA - Education, Sport, Health and Physical Activity, 1(1), 32-40. Doi: http:// hdl.handle.net/10481/48262 Recuperado de: https://goo.gl/zhxm6P

González, G., Zurita, F., Puertas, P., Espejo, T., Chacón, R. y Castro, M. (2017). Influencia de los factores sedentarios (dieta y videojuegos) sobre la obesidad en escolares de Educación Primaria. ReiDoCrea, 6 , 120-129. Recuperado de: https://goo.gl/B5jxtY

González-Hernández, J. y Portolés-Ariño, A. (2016). Recomendaciones de actividad física y su relación con el rendimiento académico en adolescentes de la Región de Murcia. Retos. Nuevas tendencias en Educación Física, Deporte y Recreación, 29, 100-104. Recuperado de: https://goo.gl/QGZsif

González-Valero, G., Zurita-Ortega, F., Puertas-Molero, P., Chacón-Cuberos, R., Espejo- Garcés, T. y Castro-Sánchez, M. (2017). Educación para la salud: implementación del programa «Sportfruits» en escolares de Granada. SPORT TK: Revista Euroamericana de Ciencias del Deporte, 6(2), 137-146. Recuperado de: https://goo.gl/ TYWGBn

Granda, J., Montilla, M., Barbero, J. C., Mingorance, A. y Alemany, I. (2010). Frecuencia de práctica y motivos de participación/no participación en actividades físicas en función del género de escolares de 10-12 años de Melilla. Revista Internacional de Ciencias del Deporte, 21(6), 280-296. Recuperado de: https://goo.gl/6AqekH

Isorna, M., Rial, R. y Vaquero, R. (2014). Motivaciones para la práctica deportiva en escolares federados y no federados. Retos: nuevas tendencias en educación física, deporte y recreación, 25, 80-84. Recuperado de: https://goo.gl/eaipB8

Klee, M. H., Tavares, R., Horta, B., Gelatti, C. y Santana, P. (2004). Prevalência e fatores associados ao sedentarismo em adolescentes de área urbana. Revista de Saúde Pública, 38(2), 157-163. Recuperado de: https://goo.gl/iN71PB

Kowalski, K., Crocker, P. y Faulkner, R. (1997). Validation of the Physical Activity Questionnaire for Older Children. Pediatric Exercise Science, 9(2), 174-186. Doi: https://doi.org/10.1123/pes.9.2.174 Recuperado de: https://goo.gl/wwxt8R

Leite, A., Carvalho de Mesquita, J. M. y Dias, A. (2015). Analysis of the causes of dissatisfaction and abandonment by clients of fitness centres. Podium-sport leisure and tourism review, 4(3), 124-139. Recuperado de: https://goo.gl/MCJCvf

Macarro, J., Romero, C. y Torres, J. (2010). Motivos de abandono de la práctica de actividad físico-deportiva en los estudiantes de Bachillerato de la provincia de Granada. Revista de Educación, 353, 495519. Recuperado de: https://goo.gl/uop89d

Marcellini, A., Perera, E., Rodhain, A. y Ferez, S. (2016). Body image and participation in physical activities by obese subjects. Sante publique, 28(1), 117-125. Recuperado de: https://goo.gl/QLhc8F

Martínez, J., Aznar, S. y Contreras, O. (2015). El recreo escolar como oportunidad de espacio y tiempo saludable. Revista Internacional de Medicina y Ciencias de la Actividad Física y el Deporte, 15(59), 419-432. Doi: http://dx.doi.org/10.15366/rimcafd2015.59.002 Recuperado de: https://goo.gl/nrgKpC

Martínez-Gómez, D., Martínez-de-Haro, V., Pozo, T., Welk, G., Villagra, A., Calle, M., et al. (2009). Fiabilidad y validez del cuestionario de actividad física PAQ-A en adolescentes españoles. Revista Española Salud Pública, 83, 427-439. Recuperado de: https://goo.gl/42H9s8

Méndez-Alonso, D., Fernández-Río, J., Méndez-Giménez, A. y PrietoSaborit, J. A. (2015). Análisis de los currículos autonómicos LOMCE de Educación Física en Educación Primaria. Retos. Nuevas tendencias en Educación Física, Deporte y Recreación, 28, 15-20. Recuperado de: https://goo.gl/YcLqiT

Merino, B. y González, E. (2006). Actividad Física y Salud en la Infancia y la Adolescencia Guía para todas las personas que participan en su educación. Madrid: Ministerio de Educación y Ciencia y Ministerio de Sanidad y Consumo. Recuperado de: https://goo.gl/BB8pfP
Molinero, O., Castro, J., Ruiz, J. R., González, J. L., Mora, J. y Márquez, S. (2010). Conductas de salud en escolares de la provincia de Cádiz. Nutrición Hospitalaria, 25(2), 280-289. Recuperado de: https://goo.gl/ VWKr4M

OMS (2010). Estrategia mundial sobre régimen alimentario, actividad física y salud. Recuperado de: https://goo.gl/QxsLp6

Oviedo, G., Sánchez, J., Castro, R., Calvo. M., Sevilla, J. C., Iglesias, A. y Guerra, M. (2013). Niveles de actividad física en población adolescente: estudio de caso. Retos: nuevas tendencias en educación física, deporte y recreación, 23, 43-47. Recuperado de: https://goo.gl/Swkv94

Palou, P., Ponseti, X., Gili, M., Borràs, P. A. y Vidal, J. (2005). Motivos para el inicio, mantenimiento y abandono de la práctica deportiva de los preadolescentes de la isla de Mallorca. Apunts. Educación física y deportes, 3(81), 5-11. Recuperado de: https://goo.gl/cm1nA4

Pastor, J. C., Gil, P., Tortosa, M. y Martínez, J. (2012). Efectos de un programa de actividad física extracurricular en niños de primer ciclo de ESO con sobrepeso y obesidad. Revista de Psicología del Deporte, 21, 379-385. Recuperado de: https://goo.gl/ZfGkyv

Piéron, M., y Ruiz-Juan, F. (2013). Influencia del ámbito familiar e iguales en los hábitos físico-deportivos de los jóvenes. Revista Internacional de Medicina y Ciencias de la Actividad Física y del Deporte, 51, 725. Recuperado de: https://goo.gl/1bLMGB

Puertas-Molero, P., González-Valero, G. y Sánchez-Zafra, M. (2017). Influencia de la práctica físico deportiva sobre la Inteligencia Emocional de los estudiantes: Una revisión sistemática. ESHPA - Education, Sport, Health and Physical Activity, 1(1), 10-24. Doi: http:// hdl.handle.net/10481/48262 Recuperado de: https://goo.gl/UFkAoj

Reverter-Masià, J., Plaza-Montero, D., Jové, M. C. y Hernández-González, V. (2014). Actividad físico-deportiva extraescolar en alumnos de primaria: el caso de Torrevieja (Alicante). Retos. Nuevas tendencias en Educación Física, Deporte y Recreación, 25, 48-52. Recuperado de: https://goo.gl/D2AyHc

Revuelta, L. y Esnaola, I. (2015). Clima familiar deportivo y autoconcepto físico en la adolescencia. European Journal of Education and Psychology, 4(1), 19-31. Recuperado de: https://goo.gl/wqGSeF

Rodríguez, P., García-Cantó, E., Sánchez-López, C. y López-Miñarro, P. (2013). Percepción de la utilidad de las clases de educación física y su relación con la práctica físico-deportiva en escolares. Cultura y Educación, 25(1), 65-76. Doi: 10.1174/113564013806309127 Recuperado de: https://goo.gl/mrmDm9

Ruiz-Risueño, J. y Ruiz-Juan, F. (2015). Actividad físico-deportiva y contexto familiar: variables predictoras de consumo de tabaco entre adolescentes españoles. Revista Iberoamericana de psicología del ejercicio y el deporte, 10(1), 121-131. Recuperado de: https://goo.gl/ 2cCRT9

Sánchez Bañuelos, F. (1996). La actividad física orientada hacia la salud. Madrid: Biblioteca Nueva.

Serra, J.R., Generelo, E. y Zaragoza, J. (2010). Barreras para la realización de actividad física en adolescentes en la provincia de Huesca. Revista Internacional de Medicina y Ciencias de la Actividad Física y el Deporte, 10(39), 470-482. Recuperado de: https://goo.gl/Mqu3JL

Ubago-Jiménez, J.L., Viciana-Garófano, V., Pérez-Cortés, A.J., MartínezMartínez, A., Padial-Ruz, R., Puertas-Molero, P. (2018). Relación entre la Teoría de las Inteligencias Múltiples y la actividad físicodeportiva. Revisión bibliográfica. Sportis Science Journal of School Sport, Physical Education and Psychomotricity, 4(1), 144-161. Doi: https://doi.org/10.17979/sportis.2018.4.1.2067 Recuperado de: https://goo.gl/2q6chF

Valdés, P., Godoy, A., Herrera, T., Álvarez, M. y Durán, S. (2014). Asociación entre estado nutricional y tiempo de actividad física escolar de niños y niñas chilenos de 4 a 14 años. Nutrición Clínica y Dietética Hospitalaria, 34(3), 57-63. Doi: 10.12873/343valdesbadilla Recuperado de: https://goo.gl/P1xoJ6

Zurita-Ortega, F. y Álvaro-González, J. (2014). Repercusión del tabaco y alcohol sobre factores académicos y familiares en adolescentes. Health and Addictions, 14(1), 59-70. Recuperado de: https://goo.gl/E9UHvt

Zurita-Ortega, F., Castro-Sánchez, M., Álvaro-González, J., RodríguezFernández, S. y Pérez-Cortés, A. J. (2016). Autoconcepto, Actividad física y Familia: Análisis de un modelo de ecuaciones estructurales. Revista de Psicología del Deporte, 25(1), 97-104. Recuperado de: https://goo.gl/CQRzdo

Zurita-Ortega, F., Cepero, M., Ruiz, L., Linares, D., Cachón, J., ZuritaMolina, F. (2011). Age and gender differences in Body Mass Index, ocular, and back disorders in 8-12-year old children. Journal of Human Sport and Exercise, 6(4), 657-672. Doi: 10.4100/jhse.2011.64.09 Recuperado de: https://goo.gl/B42t2F 